\title{
Rechtsgeschichte
}

\section{Ulrike Ludwig}

Gepflegte Streitereien und gepflegte Verträge 
in certo modo di un controllo a garanzia di validità delle stesse decisioni giudiziali, mettendo cosí i rettori e giudici forestieri al riparo dal sindacato cui erano sottoposti in uscita dalla carica (Menzinger, Milani).

Le modificazioni subite nella prassi giudiziaria dalla procedura romano-canonica - quale modello astratto di ordo iudicii retaggio della trattatistica duecentesca - tra fine del secolo XIII e inizio del successivo si connettono, oltre che ad una evoluzione dei sistemi giudiziari, alla elaborazione dottrinale e normativa della procedura sommaria. Si può osservare un'evoluzione parallela nel processo penale e in quello civile, nel senso di una razionalizzazione della procedura ordinaria ricorrendo a soluzioni offerte dallo strumento della procedura sommaria, che di per sé non costituiva un modello alternativo di processo, contrapposto all'ordinario, e non forniva uno schema processuale unico e vincolante, lasciando ampio margine di scelta ai tribunali e al loro personale giudiziario nel definire la prassi cui attenersi: lo stylus curiae. A questo ambito possono essere ricondotte anche le divergenze dall'ordo iudicii osservate nei processi civili di Marseille (Smail).

Per la sua funzionalità ai nuovi processi politico-sociali il processo penale è stato coinvolto nella crisi politica del Comune di popolo. All'inizio del Trecento si può osservare una crisi del sistema giudiziario podestarile, caratterizzata in rapporto ai processi accusatori, aventi una struttura analoga al rito civile, dalla frammentazione dell'iter procedurale, che con un incremento delle fasi "scritte « della procedura ha causato un aumento della durata complessiva dei procedimenti. La fase dibattimentale venne cosí a ridursi - come già nel rito civile - ad una serie di udienze per l'allegazione di documenti ed atti scritti da parte di procuratori e notai, che divennero cosí i veri protagonisti del processo. Ormai privato della sua valenza politica d'inizio Duecento - la città che affermava la propria iurisdictio sul territorio e sui cittadini - a metà Trecento si assiste all'affermarsi di un modello processuale divenuto ormai standard - in relazione alle generalità della applicazione dell'ordo - che in questa forma poté incontrare diffusione europea (Vallerani). Le prassi documentarie - in rapporto sia alla selettività delle registrazioni notarili, che alle strategie sociali di composizione al cui interno si collocano - rivelano i limiti e le ambiguità delle fonti giudiziarie, richiamati in chiusura del volume da Jacques Chiffoleau. Di questi limiti e aporie si dovrà tener conto in sede di valutazione storiografica e nell'adozione di adeguate strategie d'indagine per una storia "sociale « della giustizia, quale è auspicata da Claude Gauvard nelle sue conclusioni.

\section{Vincenzo Colli}

\section{Gepflegte Streitereien und gepflegte Verträge*}

Thomas Ott beschäftigt sich in seiner 2006 abgeschlossenen Dissertation mit dem albertinischen Sachsen und seinen politischen Wirkungs- kreisen im I6. Jahrhundert. Im Zentrum des Interesses steht die Frage, "was das albertinische Sachsen im Reich sausmachte`, was es 'galt‘,

\footnotetext{
* Thomas Отт, Präzedenz und Nachbarschaft. Das albertinische Sachsen und seine Zuordnung zu Kaiser und Reich im I6. Jahrhundert (Veröffentlichungen des Instituts für europäische Geschichte Mainz, Abteilung abendländische Religionsgeschichte 2I7), Mainz: Verlag Philipp von Zabern 2008, XIV, 654 S., ISBN 978-3-8053-3875-2
} 
wodurch seine Beziehungen zu anderen Ständen und zum Kaiser definiert waren « (6). Als zentrale Maßstäbe für die Einordnung Sachsens in das durch Kaiser und Reichsverband markierte Bezugsfeld werden in der Einleitung unter den Schlagworten »Präzedenz « und "Nachbarschaft " einerseits die Teilnahme an Reichsversammlungen und damit die Bedeutung von Session und Zeremoniell und andererseits das Erbeinungswesen herausgestellt. Bei Letzterem liegt der Fokus auf Erbeinungen zwischen Sachsen und Böhmen und der Frage, ob diese "langfristig für Einfluß bei Kaiser und Ständen sorgen konnten und wie sich dieser Einfluß bemaß « (27).

Die Arbeit ist in zehn Kapitel unterteilt. Im ersten Kapitel (39-5I) werden zunächst nochmals Grundlagen und Spielräume einer Hierarchie der Reichsfürsten thematisiert. In den Kapiteln II bis X werden chronologisch geordnet die Beziehungen des albertinischen Sachsens zum Reich und zu den Reichsständen anhand von Sessionsstreitigkeiten und Erbeinungen untersucht. Beginnend mit Herzog Albrecht (seit I487) bis etwa zum Regierungsantritt von Kurfürst Christian II. (I60I) wird so eine Überblicksdarstellung der Außenbeziehungen der Albertiner geboten. Abgeschlossen wird der Band mit einer Zusammenfassung und einer Schlussbetrachtung. Im Anhang finden sich schließlich neben der Transkription der "Conceptio Mauritij« (um I 546) und einem Bedenken über die Funktion und Bedeutung der Erbeinung zwischen Sachsen, Böhmen und Österreich (I620) noch eine Liste mit den Erbeinungen und Erbverbrüderungen der Wettiner mit Böhmen, Brandenburg, Henneberg und Hessen (I307-I6I 5 ). Bedauerlich ist, dass zwar ein Orts- und Personenregister, aber kein Sachregister erstellt wurde - dies hätte angesichts des Umfangs und der strikt chronologischen Anlage der Untersuchung die gezielte Rezeption auch für weiter gespannte Forschungskontexte erleichtert.

Eingeschoben in die chronologische Ordnung der Kapitel ist ein informativer Exkurs zur Bedeutung der protestatio als Mittel der Reichstagspolitik (I73-I93). Gerade der Vergleich zwischen den Protestationen in Sessionsund in Religionsfragen ist interessant: Ersteren begegnete man seit I 526 mit dem Beschluss, dass der Verhandlungsgang für Rangfragen unerheblich sei, das Verfahren also keine Präjudizien schaffe. Im Fall von religiösem Dissens wurde hingegen nicht das Verfahren in seiner Wirkmächtigkeit »neutralisiert, sondern der Reichsabschied, an den für die Protestanten nachteiligen Stellen " (192). Übergreifend kann Ott herausarbeiten, dass beide Formen der Protestation von einem neuartigen »Verständnis von Mehrheit und Minderheit, Konsens und Opposition im reichspolitischen Zusammenhang « zeugen (I9I). Letztlich wird hier der Übergang vom Postulat der Einstimmigkeit zum Mehrheitsprinzip greifbar. Innerhalb dieses Rahmens war die Protestation gerade für das Vorgehen von Minderheiten und Dissentierenden ein wichtiges Instrument für die Austragung von Konflikten und entfaltete dabei zugleich eine integrierende Wirkung - erlaubte sie doch eine Koexistenz divergierender Positionen (208f.).

Mit zahlreichen Einzelbelegen kann Ott in seiner Arbeit die Kontinuitäten, Verstetigungen und 'Vererbungen` von Rangkonflikten aufzeigen, die vor allem die Session im Fürstenrat betrafen. In einer Strukturgeschichte des Fürstenrates - die sich in verfassungs- und sozialhistorischer Hinsicht ganz wesentlich über die Fragen der Hierarchiebildung erschließe - sieht Ott dann auch eine wichtige Aufgabe künftiger Forschungen (520). Problematisch ist mitunter 
die zu enge Deutung der Sessionskonflikte. So erklärt Ott etwa, dass das albertinische Sachsen mit der Erlangung der Kurwürde nun der durch die Goldene Bulle klar geregelten Session im Kurfürstenrat unterstand. Damit besaß es keine Möglichkeit mehr, »sich auf eine ungewisse, angefochtene Session zu berufen, wann immer es von reichspolitischen Entscheidungen Abstand nehmen wollte«. Trotzdem - so Ott - ließen sich der veränderten Lage auf dem Reichstag I 547/ 48 »auch Vorzüge abgewinnen « (234f.). Eine Einschätzung, die angesichts der frisch gewonnenen Kurwürde doch etwas zu sehr im Understatement verharrt und gerade mit Blick auf das Thema der Arbeit am Eigentlichen vorbeigeht.

Neben Sessionsstreitigkeiten auf den Reichstagen bildet die Untersuchung von Erbeinungen den zweiten Schwerpunkt der Arbeit. Erbeinungen sind Nachbarschaftsabkommen, in denen sich die Vertragspartner gegenseitige Hilfe bei Eingriffen Dritter zusicherten und die im Kontext der Nachbarschaft relevanten gerichtlichen Verhältnisse und das Vorgehen bei grenzübergreifenden Irrungen regelten (23). Damit stehen sie in der Tradition der Landfrieden und unterscheiden sich deutlich von Erbverbrüderungen, in denen geregelt wurde, dass beim Aussterben eines Fürstenhauses das jeweils erbverbrüderte nachrückte.

Ott untersucht die Erbeinungen mit Blick auf die Frage, inwieweit diese Einfluss auf die Position des `Hauses Sachsen` während der Reichsversammlungen und damit letztlich auch auf Sessionsfragen hatten. Die Kombination von Sessionspolitik und Erbeinungen ist als Analyseansatz interessant, weil - wie Ott mit Verweis auf Albrecht P. Luttenberger betont - die Zugehörigkeit zu einer Erbeinung einem Reichsstand durchaus Anerkennung verschaffte, da er dadurch seine Fähigkeit zu politischem Einver- nehmen bewies. Dies wird besonders für die Allianzen im Nachgang des Augsburger Religionsfriedens betont. Inwieweit die Erbeinungen im Einzelfall auch zu einer handfesten (militärischen) Unterstützung führten, stand allerdings auf einem anderen Blatt. Gerade im ausgehenden I6. Jahrhundert wichen die Albertiner im Falle der von Böhmen eingeforderten militärischen Unterstützung eher auf die Bewilligung von Reichs- und Kreishilfen aus und griffen damit auf ein politisches Mittel zurück, das sich im Vergleich mit der Erbeinung auch als Quelle der Reputation eignete - da die Hilfe hier kollektiv und vor allem freiwillig geleistet wurde.

Als zentrales Ergebnis betont Ott, dass das albertinische Sachsen im Laufe des I6. Jahrhunderts eine Position »bedingter Distanz zu den Reichsorganen « entwickelte. Zugleich hätten die Albertiner innerhalb der Reichsorgane eine Vormachtstellung errungen, die sie bis zum Ende des I6. Jahrhunderts behaupten konnten (507). Für den Aufbau dieser Position werden viele mögliche Faktoren benannt, etwa die regionale Lage, der Bekenntnisstand, die Kontakte zum Kaiserhof und zu den territorialen Nachbarn, der Aufstieg zum Kurfürstentum, der Einsatz ökonomischer Ressourcen usw. usf.

In dieser Präsentation des Ertrages zeigen sich exemplarisch zwei übergreifende Probleme dieser Arbeit: So ist einerseits festzustellen, dass die vorgestellten Beobachtungen im Einzelnen durchaus richtig sind, aber es fehlt die Gewichtung, der Mut zur Akzentuierung angesichts der erforschten Komplexität. Andererseits verschenkt Ott durch die mitunter im Unklaren bleibende Begrifflichkeit Chancen auf eine präzisere Thesenbildung. Denn - um den eben angeführten Verweis zu nutzen - was eine bedingte Distanz angesichts der errungenen Vormachtstellung meint ist unsicher. Und wieso - so die 
abschließende These Otts - von einer »selbstgewählten `Zuordnung « « des albertinischen Sachsen zum Reich auszugehen sei und nicht von einer »Integration «, bleibt ohne eine Konzeptionalisierung der Begriffe letztlich offen. Gleichwohl ist zu betonen, dass Ott mit der vorliegenden Studie einen Beitrag auf dem Weg hin zu

\section{Oberflächlich *}

Bei dem vorliegenden Buch handelt es sich um eine aus dem Trierer Graduiertenkolleg »Sklaverei, Knechtschaft und Frondienst, Zwangsarbeit « hervorgegangene juristische Dissertation. Behandelt wird in Frankes Studie die Stellung von insgesamt fünf Naturrechtsphilosophen (Francisco Suárez, Hugo Grotius, Thomas Hobbes, Samuel von Pufendorf und John Locke) zur Frage der Sklaverei und der Unfreiheit. Die leitende Frage der Studie lautet, ob die ausgewählten Autoren die Sklaverei entweder naturrechtlich $\mathrm{zu}$ legitimieren versuchten oder als naturrechtswidrig betrachteten, ob die »Naturrechtsphilosophen des I7. Jahrhunderts « die Abschaffung der Sklaverei forderten oder »in der Unfreiheit eine hinnehmbare Institution " sahen (IO). Die Antwort auf diese Frage sei von "allergrößte[m] Interesse ", unter anderem deshalb, weil insbesondere Hobbes und Locke als "geistige Väter der Aufklärung " anzusehen seien. Die Beschränkung auf die genannten Autoren des I7. Jahrhunderts wird nicht näher begründet. Dabei liegt auf der Hand, dass diese Beschränkung dazu führt, dass die Studie insgesamt wenig ergiebig ist, weil die Rechtstheorien der ausgewählten Autoren (von Locke einmal abgesehen) - wie Franke selbst in Bezug auf einer landesgeschichtlichen Neukonzeptionalisierung der Verfassungsgeschichte des Alten Reiches liefert und für Sachsen eine Lücke in der Forschung zu schließen vermag.

\section{Ulrike Ludwig}

Pufendorf feststellt - die rechtliche Freiheit des Einzelnen für ein »weitgehend disponibles Rechtsgut « halten (250). Die rechtliche Selbstversklavung stellt für sie somit kein juridisches Problem dar. Ertragreicher wäre es gewesen, die Studie auf das I8. Jahrhundert auszudehnen, weil nun die schon bei Locke zu findenden Einwände gegen die rechtliche Möglichkeit der Sklaverei systematisch ausgebaut werden (insbesondere bei Montesquieu und Rousseau) und die Sklaverei zugleich Gegenstand historischer und soziologischer Studien (Millar, Reitemeier) wird. Die Frage, warum sich die Einstellung zur Legitimität der Sklaverei im Laufe des I8. Jahrhunderts ändert und sie deshalb für Autoren wie Montesquieu, Rousseau, Raynal oder Kant naturrechtswidrig ist, warum außerdem eine intensive Debatte über die Aufhebung der Sklaverei und vor allem des Sklavenhandels in Gang kommt - diese Fragen werden von Franke nicht gestellt.

Franke kommt in einer "Gesamtzusammenfassung « (309 ff.) zu dem Ergebnis, dass durch seine Studie die Annahme zu Fall gebracht würde, »das Naturrecht habe im Verlauf des I7. Jahrhunderts einen Entwicklungsprozess durchlaufen, an dessen Ende die vollständige

\footnotetext{
* Bernd Franke, Sklaverei und Unfreiheit im Naturrecht des I7. Jahrhunderts (Sklaverei Knechtschaft - Zwangsarbeit. Untersuchungen zur Sozial-, Rechts- und Kulturgeschichte 5), Hildesheim, Zürich, New York: Olms Verlag 2009, IX, 337 S., ISBN 978-3-487-I37I9-3
} 